\title{
Significance of Toll-like Receptor 4 ( TLR4 )\& Liver Fatty Acid- binding Protein (L-FABP) Expression in Hepatocellular Carcinoma An Immunohistochemical Study
}

Eman El-Gendy ${ }^{\text {a }}$, Reda El badawy ${ }^{\text {b }}$, Ranih Zakaria ${ }^{\text {a }}$, Taghreed Abd Elsamee ${ }^{\text {a }}$, Hala A. Agina ${ }^{\text {a }}$

a Department of Pathology, Benha faculty of medicine, Benha University, Egypt. b Department of Hepatology, Gastroentrology and Infectious Diseases, Benha faculty of medicine, Benha University, Egypt

Correspondence to: Eman El-Gendy, Department of pathology, Benha faculty of medicine, Benha University, Egypt.

\section{Email:}

monyese2020@yahoo.com

Received: 5 December 2020

Accepted: 9 December 2020

\begin{abstract}
:
Background: Persistent inflammatory conditions can induce tumorigenesis . It is therefore very important to explore the molecular mechanisms involved in progression from chronic hepatitis to cirrhosis to HCC - to identify factors useful for the development of effective therapies that improve patient survival. Aim of the study: Evaluation of TLR-4 and L-FABP expression in cases of chronic hepatitis, cirrhosis and HCC and correlate them with clinicopathological parameters. Methods: A retrospective, immunohistochemical study was performed on 50 liver cases; 32 cases of HCC, 10 cases of chronic hepatitis $\mathrm{C}$, and 8 cases of cirrhosis. Results: There was statistical significant increase in TLR-4 expression in $\mathrm{HCC}$ than both chronic liver hepatitis and cirrhosis $(\mathrm{P}<0.05)$. There was positive statistical significant correlation between TLR-4 expression and size \& grade of HCC $(\mathrm{P}<0.05)$. There was highly statistical significant decrease in L-FABP expression in HCC than both chronic liver hepatitis and cirrhosis $(\mathrm{P}<$
\end{abstract} 0.001). Conclusion: TLR-4 has a role in hepatocarcinogenesis as TLR-4 expression increased with progression from chronic hepatitis to cirrhosis to HCC. Increased TLR-4 expression associated with large tumor size and higher grade of HCC, so it may serve a tool for prognosis of HCC. L-FABP expression decreased with progression from chronic hepatitis to cirrhosis to HCC. So its downregulation may contribute in pathogenesis of HCC.

Key words: TLR-4, L-FABP, Hepatocellular carcinoma, chronic hepatitis C.

Abbreviations: Hepatocellular carcinoma (HCC), Toll-like receptor 4(TLR4), Liver-Fatty acid-binding proteins (L-FABP). 



\section{Introduction}

Hepatocellular carcinoma (HCC) is the most common primary liver cancer globally, it is the sixth most common cancer and the third leading cause of cancer related death (1).

In Egypt, HCC is the fourth most common cancer and is the second cause of cancer mortality in both sexes. HCC contributes to $14.8 \%$ of all cancer mortality in Egypt (2).

Hepatocellular carcinoma (HCC) is a severe health problem worldwide, as it is characterized by a high rate of recurrence and poor prognosis. Despite various treatment options, HCC is associated with a high mortality rate and remains an intractable illness (3)

Persistent inflammatory conditions can induce tumorigenesis. Hepatocellular carcinoma (HCC) is closely associated with chronic inflammatory liver diseases. . It is therefore very important to further explore the molecular mechanisms involved in progression from chronic hepatitis to cirrhosis to HCC to identify factors useful for the development of effective therapies that improve patient survival (4).
Toll-like receptors (TLRs) are a family of pattern-recognition receptors that play a critical role in the activation of the innate immune system by recognizing pathogenassociated molecular patterns (PAMPs) (5)

Toll-like receptor 4(TLR4) is one of the most intensively studied members of the TLR family. It is also expressed in several liver cells, such as hepatocytes, Kupffer cells, and hepatic stellate cells, which are associated with liver disease. Under normal circumstances, the expression of TLR4 is at a relatively low level, however, when the liver is damaged under pro-inflammatory conditions, TLR4 expression is upregulated (6).

To date experimental studies, show that TLR4 is expressed on several types of tumor cells and signaling via TLR4 may play an important role in carcinogenesis, metastasis and cancer progression. Lipopolysaccharides (LPS), an agonist of TLR4, induces the interaction of TLR4 with adaptor molecule MyD88, which in turn activates downstream NF-kB signaling pathways subsequently causing inflammatory mediator production and promotion of HCC cell survival and 
Fatty acid-binding proteins (FABPs) bind and sequester potentially toxic long-chain fatty acids in the cytosol so that they may be rapidly removed via oxidative or storage organelles (8)

Liver-FABP (L-FABP or FABP1) is the first of the FABPs to be described so far. It is expressed in very high levels in liver, intestine and kidneys. FABP1 is positively regulated by hepatocyte nuclear factor 1 $(\mathrm{HNF} 1 \alpha)$. While the downregulation of LFABP expression is critically important in the diagnostic classification of HCA, and is correlated with various clinicopathological features, the significance of downregulation of LFABP expression in $\mathrm{HCC}$ is largely unknown (9)

The present study aimed at evaluation of TLR-4 and L-FABP expression in chronic hepatitis, cirrhosis and HCC using immunohistochemical staining and related their expression with clinicopathological data.

\section{Material \& Methods}

\section{Study groups:}

A retrospective study was performed on paraffin sections of 50 specimens of liver tissues including 10 specimens of chronic hepatitis $\mathrm{C}$, and 8 specimens of cirrhosis and 32 specimens of HCC. In addition 6 specimens of normal liver core biopsy obtained from donors for liver transplantation were used as a control group. They were obtained from the departments of pathology, Faculty of Medicine, Benha University and National Liver Institute, Menofia University, during the period between March, 2010 and September, 2018. This research plan was approved by the Research Ethics Committee of Faculty of Medicine, Benha University, Egypt.

The following data were collected from the patient files: age, gender, alpha fetoprotein level (when available), presence or absence of hepatitis viral infection (HCV-RNA and HBV-DNA) by quantitative polymerase chain reaction and radiologic findings (Tumour focality, site and size). All sections subjected to: Hematoxylin and eosin stain for histopathological assessment to confirm diagnosis and immunohistochemical staining for evaluation of TLR-4 and LFABP expression.

\section{Histopathological evaluation:}

From each representative paraffin block of the studied cases, 4- $\mu \mathrm{m}$ thick sections 
were cut, stained by haematoxylin and eosin $(\mathrm{H} \& \mathrm{E})$ and re-evaluated to confirm the diagnosis and to assess the following: for chronic hepatitis cases (the grade of activity and stage of fibrosis using Metavir scoring system) (10), for HCC ( type (11), grade According to WHO classification of tumors of digestive system (12), (High grades HCC confirmed to be HCC by positivity to HepPar-1 and Arginase-1), stage according to American Joint Committee on Cancer (AJCC) uses tumor-nodemetastasis (TNM) system $8^{\text {th }}$ edition (13) and vascular invasion ).

\section{Immunohistochemical evaluation}

Immunohistochemical (IHC) procedure was performed according to manufacturer's instructions, using polyclonal Anti-TLR4 antibody (Cat. \# YPA1054- Chongqing Biospes Co.,Ltd ,China) (50ul) and polyclonal AntiLFABP antibody (Cat. \#YPA1296Chongqing Biospes Co.,Ltd ,China) (0.1 $\mathrm{ml} \mathrm{).} \mathrm{at} \mathrm{a} \mathrm{dilution} \mathrm{of} \mathrm{1:50,} \mathrm{at} \mathrm{room}$ temperature overnight. Immunodetection was carried out using a standard labeled streptavidin-biotin system (Genemed, CA 94080, USA, South San Francisco). Antigen retrieval was done by using 10 $\mathrm{mmol} / \mathrm{L}$ citrate monohydrate buffer $(\mathrm{pH}$
6.0) and heating for 15 minutes in the microwave. Freshly prepared chromogen diaminobenzine (DAB, Envision ${ }^{\mathrm{TM}}$ Flex /HRP-Dako, REF K 8000) was used.

\section{Negative \& positive controls:}

Normal gastric mucosa used as a positive control for TLR4 (14), and normal intestinal mucosa used as a positive control For L-FABP (15).

For negative controls, the primary antibody was replaced with a solution of BSA in phosphate-buffered saline (PBS).

\section{Immunohistochemical assessment:}

TLR4: positivity was considered when there was brownish cytoplasmic staining .The extent of TLR4 positive hepatocytes was assessed by evaluating the entirety of the tissue on each stained slide into: 1 , < $25 \%$ of hepatocytes positive for TLR4; 2 , $25 \%-75 \%$ of hepatocytes positive for TLR4; 3, >75\% of hepatocytes positive for TLR4 (16).

L-FABP: positivity was considered when there was brownish cytoplasmic staining interpreted the staining results, which were analyzed for the intensity and percentage of staining area by using Quick-score analysis, whereby scores (Q) were calculated as follows: $\mathrm{Q}=$ Percentage of positive cells $(\mathrm{P}) \times$ Intensity 

(I); maximum $\mathrm{Q}=300$. The results were then graded according to the following criteria:1: $\mathrm{Q}=0-99$, weak staining; $2: \mathrm{Q}=$ 100-199, moderate staining; 3: Q = 200300, strong staining (17) .

Statistical analysis: Data were collected, tabulated and statistically analyzed using a personal computer with SPSS version 20, p value is Statistically significant when $\leq 0.05$. Receiver-operating characteristic (ROC) curve was used to predict sensitivity, specificity and accuracy of immunohistochemical score.

\section{Results}

Histopathological examination of 50 liver cases showed $32(64 \%)$ cases of Hepatocellular carcinoma (HCC), $10(20 \%)$ cases of chronic hepatitis $\mathrm{C}$, and $8(16 \%)$ cases of cirrhosis. In addition to 6 cases of normal liver tissues as a control group.

\section{Clinicopathological results}

Table (1) : Scoring of TLR-4 in chronic hepatitis C cases regarding activity

\begin{tabular}{|c|c|c|c|c|c|c|c|c|}
\hline \multirow[b]{2}{*}{ TLR4 } & \multicolumn{2}{|c|}{$1+(n=4)$} & \multicolumn{2}{|c|}{$2+(n=4)$} & \multicolumn{2}{|c|}{$3+(n=2)$} & \multirow{2}{*}{$\begin{array}{l}\text { Statistical } \\
\text { test (FET) }\end{array}$} & \multirow[t]{2}{*}{$P$ value } \\
\hline & No & $\%$ & No & $\%$ & No & $\%$ & & \\
\hline \multicolumn{9}{|c|}{ Activity grades } \\
\hline A1 & 4 & 100 & 0 & 0.0 & 0 & 0.0 & 10.88 & $0.004 * *$ \\
\hline A2 & 0 & 0.0 & 3 & 75.0 & 0 & 0.0 & & \\
\hline A3 & 0 & 0.0 & 1 & 25.0 & 2 & 100 & & \\
\hline
\end{tabular}

TLR-4=Toll like receptor-4, n=number, FET=Fisher exact test , P=probability 
Table (2): Relation between TLR-4 expression and clinicohistopathological data of HCC

\begin{tabular}{|c|c|c|c|c|c|c|c|c|}
\hline \multirow[b]{2}{*}{ TLR4 } & \multicolumn{2}{|c|}{$1+(n=1)$} & \multicolumn{2}{|c|}{$2+(n=16)$} & \multicolumn{2}{|c|}{$3+(n=15)$} & \multirow{2}{*}{$\begin{array}{l}\text { Statistical } \\
\text { test (FET) }\end{array}$} & \multirow[t]{2}{*}{$P$ value } \\
\hline & No & $\%$ & No & $\%$ & No & $\%$ & & \\
\hline \multicolumn{9}{|l|}{$\overline{\text { Age (yrs) }}$} \\
\hline Median (IQR) & \multicolumn{2}{|c|}{$56.0(56.0-56.0)$} & \multicolumn{2}{|c|}{$55.0(47.2-60.7)$} & \multicolumn{2}{|c|}{$56.0(54.0-67.0)$} & $\mathrm{KW}=1.6$ & 0.45 \\
\hline \multicolumn{9}{|l|}{ Sex } \\
\hline Male & 1 & 100 & 12 & 75.0 & 12 & 80.0 & 0.65 & 1.0 \\
\hline Female & 0 & 0.0 & 4 & 25.0 & 3 & 20.0 & & \\
\hline \multicolumn{9}{|l|}{ Aetiology } \\
\hline $\mathrm{HCV}$ & 1 & 100 & 15 & 93.8 & 15 & 100 & 2.69 & 1.0 \\
\hline HBV & 0 & 0.0 & 1 & 6.3 & 0 & 0.0 & & \\
\hline AFP Median (IQR) & & 300 & $335(250$ & $37.5)$ & $600(3$ & $1000)$ & $\mathrm{KW}=3.95$ & 0.14 \\
\hline \multicolumn{9}{|l|}{ Focal lesion } \\
\hline Single & 1 & 100 & 13 & 81.3 & 13 & 86.7 & 3.54 & 0.26 \\
\hline Multiple & 0 & 0.0 & 3 & 18.8 & 2 & 13.3 & & \\
\hline \multicolumn{9}{|l|}{ Site } \\
\hline Rt lobe & 1 & 100 & 8 & 50.0 & 8 & 53.3 & 2.42 & 0.88 \\
\hline Lt lobe & 0 & 0.0 & 7 & 43.8 & 5 & 33.3 & & \\
\hline Both & 0 & 0.0 & 1 & 6.3 & 2 & 13.3 & & \\
\hline Size Median (IQR) & & 2.0 & $3.0(2$ & $-4.5)$ & & $5-6.0)$ & $\mathrm{KW}=7.49$ & $0.024 *$ \\
\hline \multicolumn{9}{|l|}{ Histopathological pattern } \\
\hline Clear & 0 & 0.0 & 3 & 18.8 & 0 & 0.0 & 13.55 & $0.01 *$ \\
\hline Solid & 0 & 0.0 & 0 & 0.0 & 6 & 40.0 & & \\
\hline Trabecular & 0 & 0.0 & 10 & 62.5 & 6 & 40.0 & & \\
\hline Trab+acina & 1 & 100 & 3 & 18.8 & 3 & 20.0 & & \\
\hline \multicolumn{9}{|l|}{ Grade } \\
\hline Well differentiated & 0 & 0.0 & 5 & 31.2 & 1 & 6.7 & 10.76 & $0.048 *$ \\
\hline Moderate differentiated & 1 & 100 & 8 & 50.0 & 4 & 26.7 & & \\
\hline Poorly differentiated & 0 & 0.0 & 3 & 18.8 & 6 & 40.0 & & \\
\hline Undifferentiated & 0 & 0.0 & 0 & 0.0 & 4 & 26.7 & & \\
\hline \multicolumn{9}{|l|}{ Grade } \\
\hline Low grade & 1 & 100 & 13 & 81.2 & 5 & 33.3 & 7.82 & $0.01 *$ \\
\hline High grade & 0 & 0.0 & 3 & 18.8 & 10 & 66.7 & & \\
\hline \multicolumn{9}{|l|}{ Vascular invasion } \\
\hline Yes & 1 & 100 & 5 & 31.3 & 8 & 53.3 & 2.75 & 0.21 \\
\hline No & 0 & 0.0 & 11 & 68.8 & 7 & 46.7 & & \\
\hline \multicolumn{9}{|l|}{ Stage } \\
\hline T1 & 0 & 0.0 & 4 & 25.0 & 1 & 6.7 & 7.89 & 0.23 \\
\hline $\mathbf{T} 2$ & 0 & 0.0 & 6 & 37.5 & 5 & 33.3 & & \\
\hline T3 & 0 & 0.0 & 5 & 31.3 & 8 & 53.3 & & \\
\hline T4 & 1 & 100 & 1 & 6.3 & 1 & 6.7 & & \\
\hline \multicolumn{9}{|l|}{ Stage } \\
\hline Early stage & 0 & 0.0 & 10 & 62.5 & 6 & 40.0 & 2.50 & 0.29 \\
\hline Advanced stage & 1 & 100 & 6 & 37.5 & 9 & 60.0 & & \\
\hline \multicolumn{9}{|l|}{ L-FABP } \\
\hline $1+$ & 1 & 100 & 5 & 31.3 & 11 & 73.3 & 10.22 & $0.018 *$ \\
\hline $2+$ & 0 & 0.0 & 11 & 68.8 & 3 & 20.0 & & \\
\hline $3+$ & 0 & 0.0 & 0 & 0.0 & 1 & 6.7 & & \\
\hline
\end{tabular}

TLR-4=Toll like receptor-4, $\mathrm{n}=$ Number, , AFP= Alpha fetoprotein, HCC= Hepatocellular carcinoma, FET=Fisher Exact test, $\mathrm{P}=$ probability, $\mathrm{KW}=$ Kruskal Wallis. 
Table (3) ): Comparison between and chronic hepatitis C, cirrhosis and HCC groups regarding TLR-4 expression

\begin{tabular}{|c|c|c|c|c|c|c|c|c|}
\hline \multirow{2}{*}{$\begin{array}{l}\text { TLR4 } \\
\text { expression }\end{array}$} & \multicolumn{2}{|c|}{ HCC (32) } & \multicolumn{2}{|c|}{ Cirrhosis (8) } & \multicolumn{2}{|c|}{ Hepatitis (10) } & \multirow{2}{*}{$\begin{array}{l}\text { Statistical } \\
\text { test (FET) }\end{array}$} & \multirow[t]{2}{*}{$P$ value } \\
\hline & No & $\%$ & No & $\%$ & No & $\%$ & & \\
\hline Score 1+ & 1 & 3.1 & 0 & 0.0 & 4 & 40.0 & 9.57 & $0.015^{*}$ \\
\hline Score 2+ & 16 & 50.0 & 2 & 25.0 & 4 & 40.0 & & \\
\hline Score 3+ & 15 & 46.9 & 6 & 75.0 & 2 & 20.0 & & \\
\hline
\end{tabular}

$\mathrm{N}=$ Number , TLR-4=Toll like receptor-4, HCC=hepatocellular carcinoma, FET=Fisher exact test, , P=probability
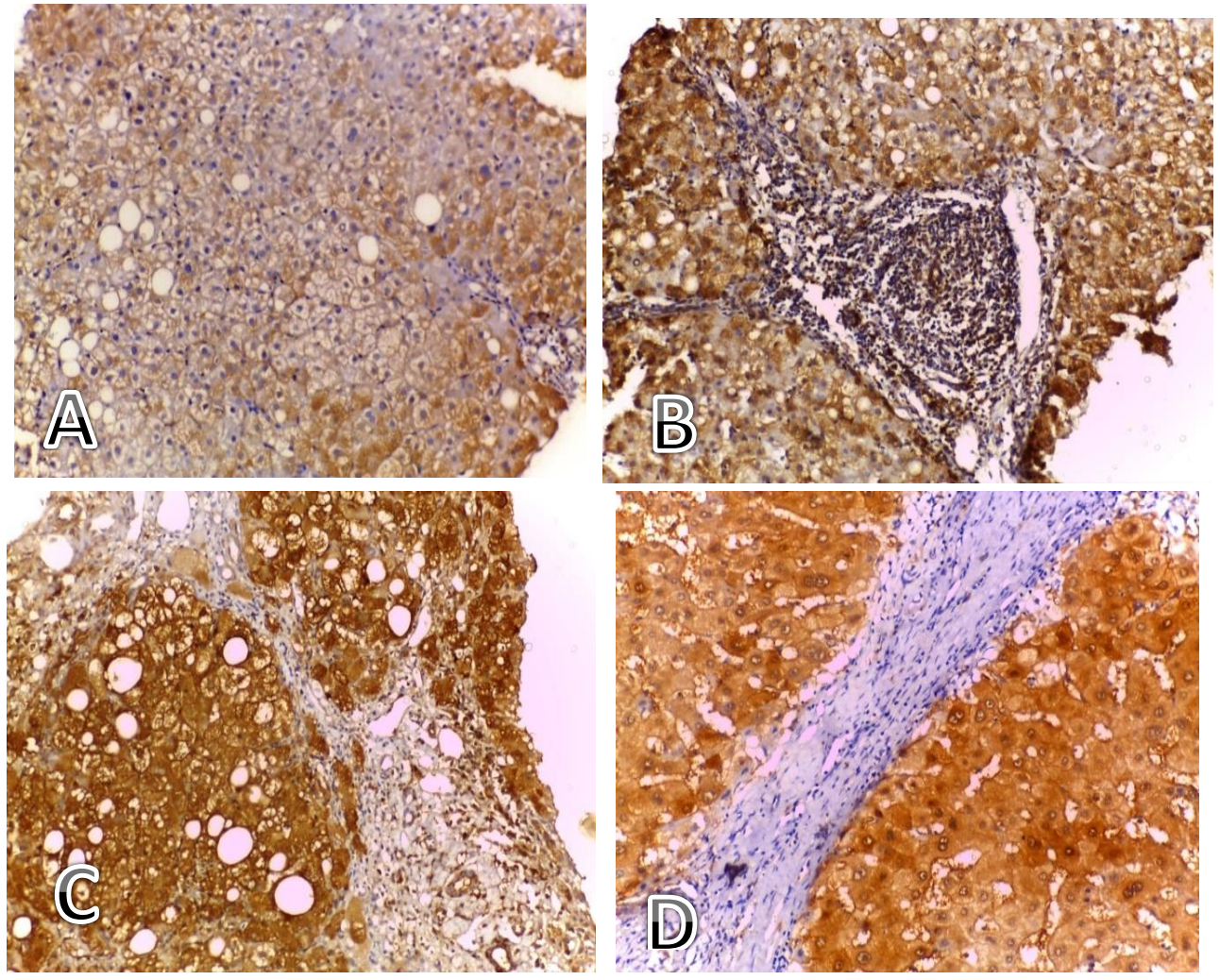

Figure (1 ): A) case of chronic hepatitis with mild activity (A1)and mild fibrosis(F1) showing positive cytoplasmic TLR-4 expression score (1+) (IHC x200).B) case of chronic hepatitis with moderate activity (A2)and moderate fibrosis(F2) showing positive cytoplasmic TLR-4 expression score (2+) (IHC x200).C) case of chronic hepatitis with sever activity (A3)and fibrosis(F3) showing positive cytoplasmic TLR-4 expression score (3+) (IHC x200).D) case of cirrhosis showing positive cytoplasmic TLR-4 expression score (3+) (IHC X200) 

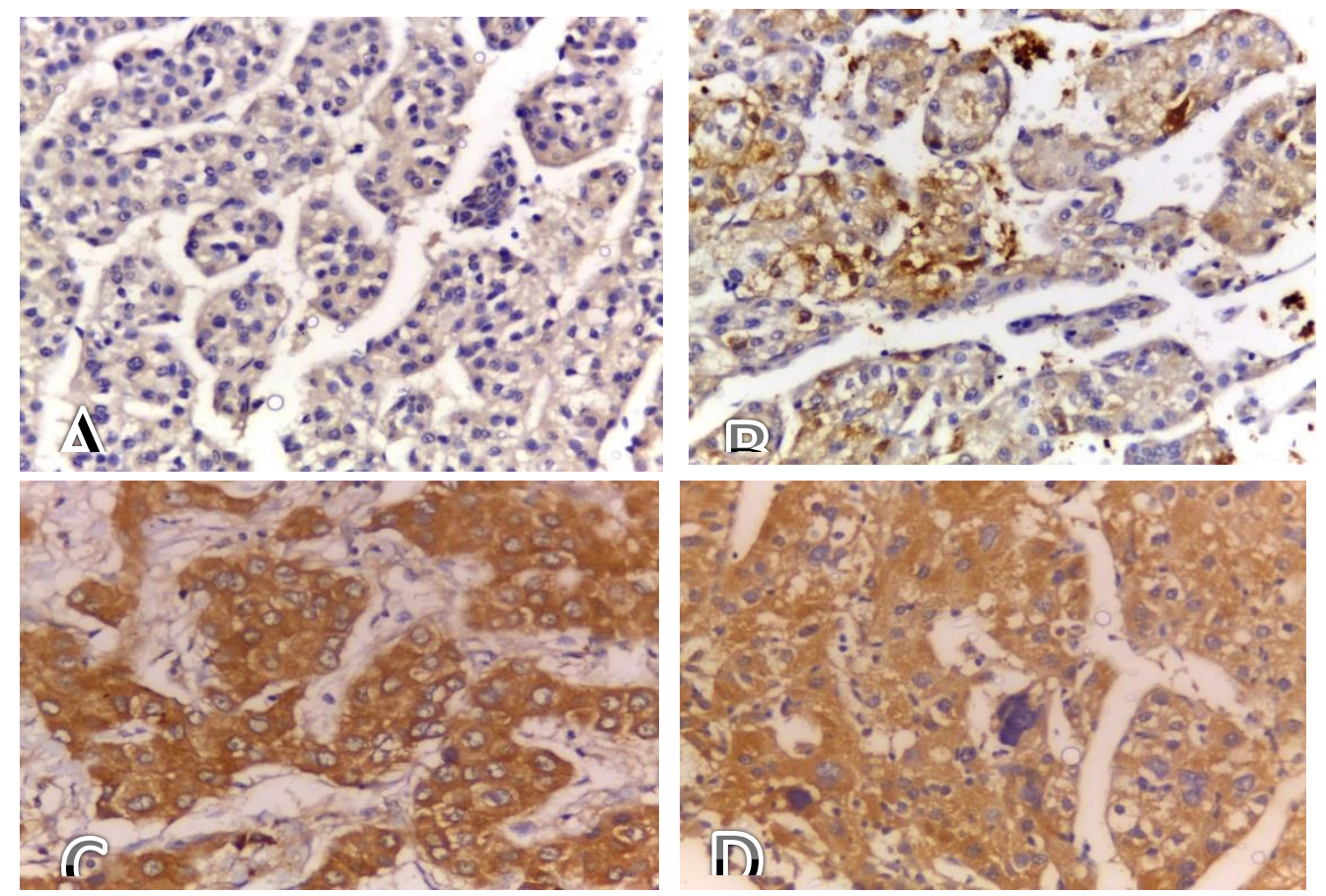

Figure(2):A) case of well differentiated HCC showing cytoplasmic TLR-4 expression score (2+) (IHC $\mathbf{x 4 0 0}$ ),B) case of moderately differentiated HCC with cytoplasmic TLR-4 expression score (2+) (IHC $\mathbf{x 4 0 0}$ ),C) case of poorly differentiated hepatocellular carcinoma showing cytoplasmic TLR-4 expression score (3+) (IHC x400),D) case of undifferentiated hepatocellular carcinoma showing cytoplasmic TLR-4 expression score (3+) (IHC x400)

\section{L-FABP expression:}

-All cases of studied normal group were scored $(+3)$.

- There was highly statistical significant decrease in L-FABP expression with progression from chronic hepatitis to cirrhosis to HCC ( $p<0.001)$, table (5), figure (3\&4).

-There was no statistical significant relation between L-FABP expression and clinicopathological parameters of HCC table (4).

\section{Correlation between TLR4 and L- FABP:}

-There was significant inverse relation between TLR4 and L-FABP regarding their expression in chronic hepatitis $\mathrm{C}$, cirrhosis and HCC group $(\mathrm{P}<0.05)$. Table (6). 
Table (4) ):Relation between L-FABP expression and clinicohisto pathological data of HCC

\begin{tabular}{|c|c|c|c|c|c|c|c|c|}
\hline \multirow{2}{*}{$\begin{array}{l}\text { L-FABP } \\
\text { HCC } \\
\end{array}$} & \multicolumn{2}{|c|}{$1+(n=17)$} & \multicolumn{3}{|c|}{$2+(n=14)$} & \multirow{2}{*}{$\begin{array}{r}3+(n=1) \\
\%\end{array}$} & \multirow{2}{*}{$\begin{array}{l}\text { Statistical } \\
\text { test (FET) }\end{array}$} & \multirow[t]{2}{*}{$P$ value } \\
\hline & No & $\%$ & No & $\%$ & No & & & \\
\hline \multicolumn{9}{|l|}{ Age (yrs) } \\
\hline Median (IQR) & \multicolumn{2}{|c|}{$56(54.5-65)$} & \multirow{2}{*}{\multicolumn{3}{|c|}{$53(44-62)$}} & $55(55-55)$ & $\mathrm{KW}=3.09$ & 0.21 \\
\hline \multicolumn{6}{|l|}{ Sex } & & & \\
\hline Male & 12 & 70.6 & 12 & 85.7 & 1 & 100 & \multirow[t]{3}{*}{1.43} & \multirow[t]{3}{*}{0.54} \\
\hline Female & 5 & 29.4 & 2 & 14.3 & 0 & 0.0 & & \\
\hline \multicolumn{7}{|l|}{ Aetiology } & & \\
\hline $\mathrm{HCV}$ & 17 & 100 & 13 & 92.9 & 1 & 100 & 2.97 & 0.47 \\
\hline HBV & 0 & 0.0 & 1 & 7.1 & 0 & 0.0 & & \\
\hline AFP Median (IQR) & \multicolumn{2}{|c|}{$450(315-1000)$} & \multicolumn{2}{|c|}{$300(237.5-562.5)$} & & 500 & $\mathrm{KW}=3.34$ & 0.19 \\
\hline \multicolumn{9}{|l|}{ Focal lesion } \\
\hline Single & 15 & 88.2 & 10 & 71.4 & 1 & 100 & \multirow[t]{2}{*}{1.87} & \multirow[t]{2}{*}{0.49} \\
\hline Multiple & 2 & 11.8 & 4 & 28.6 & 0 & 0.0 & & \\
\hline \multicolumn{9}{|l|}{ Site } \\
\hline Rt lobe & 8 & 47.1 & 8 & 57.1 & 1 & 100 & \multirow[t]{3}{*}{3.12} & \multirow[t]{3}{*}{0.73} \\
\hline Lt lobe & 8 & 47.1 & 4 & 28.6 & 0 & 0.0 & & \\
\hline Both & 1 & 5.9 & 2 & 14.3 & 0 & 0.0 & & \\
\hline Size $\quad$ Median (IQR) & \multicolumn{2}{|c|}{$4.5(3.0-6.5)$} & \multicolumn{2}{|c|}{$3.5(2.38-4.75)$} & & 3.0 & $K W=2.18$ & 0.34 \\
\hline Histopathology & & & & & & & & \\
\hline Clear & 1 & 5.9 & 2 & 14.3 & 0 & 0.0 & 5.21 & 0.63 \\
\hline Solid & 5 & 29.4 & 1 & 7.1 & 0 & 0.0 & & \\
\hline Trabecular & 8 & 47.1 & 7 & 50.0 & 1 & 100 & & \\
\hline Trab+acina & 3 & 17.6 & 4 & 28.6 & 0 & 0.0 & & \\
\hline Grade & & & & & & & & \\
\hline Well differentiated & 2 & 11.8 & 4 & 28.6 & 0 & 0.0 & 6.65 & 0.36 \\
\hline Mod. differentiated & 6 & 35.3 & 6 & 42.9 & 1 & 100 & & \\
\hline Poorly differentiated & 5 & 29.4 & 4 & 28.6 & 0 & 0.0 & & \\
\hline Undifferentiated & 4 & 23.5 & 0 & 0.0 & 0 & 0.0 & & \\
\hline Grade & & & & & & & & \\
\hline Low grade & 8 & 47.1 & 10 & 71.4 & 1 & 100 & 2.48 & 0.35 \\
\hline High grade & 9 & 52.9 & 4 & 28.6 & 0 & 0.0 & & \\
\hline Vascular inv & & & & & & & & \\
\hline Yes & 10 & 58.8 & 4 & 28.6 & 0 & 0.0 & 3.5 & 0.15 \\
\hline No & 7 & 41.2 & 10 & 71.4 & 1 & 100 & & \\
\hline Stage & & & & & & & & \\
\hline T1 & 2 & 11.8 & 3 & 21.4 & 0 & 0.0 & 7.46 & 0.29 \\
\hline T2 & 4 & 23.5 & 6 & 42.9 & 1 & 100 & & \\
\hline T3 & 10 & 58.8 & 3 & 21.4 & 0 & 0.0 & & \\
\hline T4 & 1 & 5.9 & 2 & 14.3 & 0 & 0.0 & & \\
\hline Stage & & & & & & & & \\
\hline Early stage & 6 & 35.3 & 9 & 64.3 & 1 & 100 & 3.47 & 0.16 \\
\hline Advanced stage & 11 & 64.7 & 5 & 35.7 & 0 & 0.0 & & \\
\hline
\end{tabular}

L-FABP=Liver- fatty acid binding protein, $\mathrm{n}=$ Number, $\mathrm{AFP}=$ Alpha fetoprotein, $\mathrm{HCC}=$ Hepatocellular carcinoma, FET=Fisher Exact test, $\mathrm{P}=$ probability, $\mathrm{KW}=$ Kruskal Wallis.. 
Table (5): Comparison between chronic hepatitis C, cirrhosis and HCC groups regarding L-FABP expression

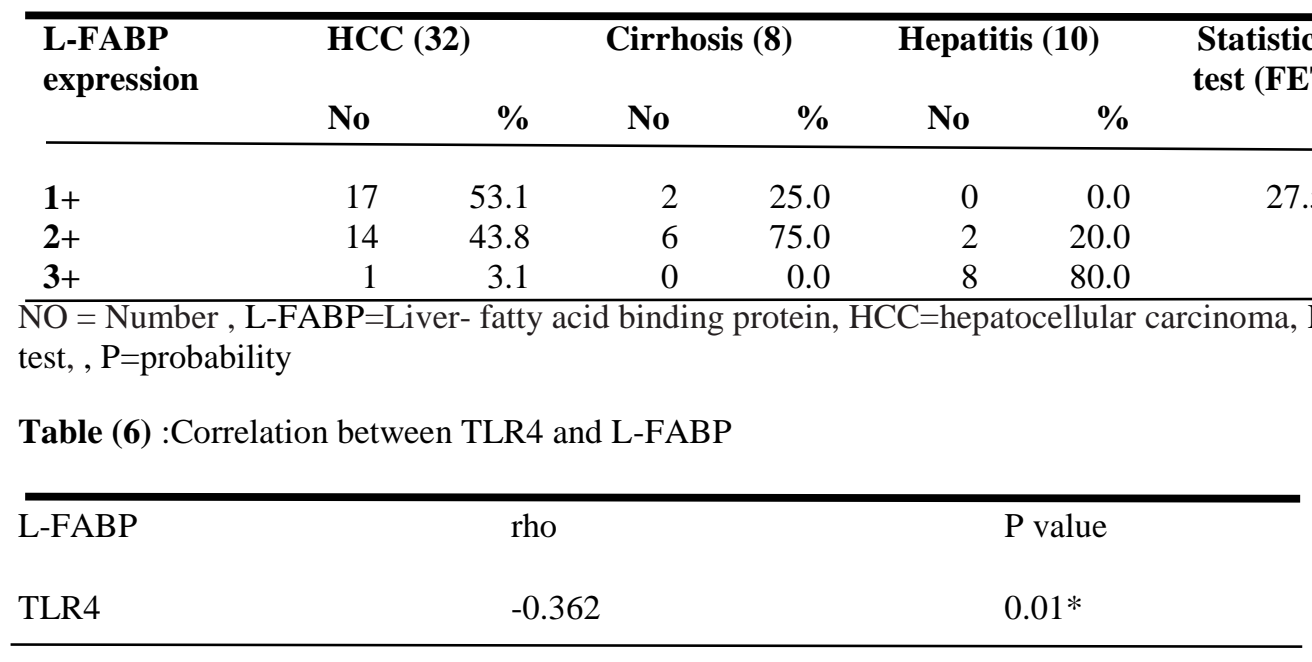

\section{ROC curve results:}

Receiver-operating characteristic (ROC) curve was used to predict sensitivity, specificity and accuracy of TLR4 \& LFABP immunohistochemical score in hepatitis, Cirrhosis and HCC groups. Both TLR4 \& L-FABP were equally in accuracy to differentiate HCC from Cirrhosis , Sensitivity was $53.1 \%$, specificity was $75.0 \%$ and accuracy was $57.5 \%$ as shown in table $(\mathbf{7 , 9 )}$. While LFABP was more accurate than TLR4 to predict hepatitis from Cirrhosis, accuracy was (88.9\% vs $22.2 \%)$ as shown in table $(8,10)$.

Receiver-operating characteristic (ROC) curve was used to predict sensitivity, specificity and accuracy of TLR4 \& LFABP immunohistochemical score to detect high grade HCC from low grade HCC. TLR4 was more accurate than LFABP to detect high grade HCC from low grade HCC, accuracy was $(75.0 \%$ vs $62.5 \%)$ as shown in table $(\mathbf{1 1}, \mathbf{1 2})$. 
Table (7) : Validity of TLR4 to predict HCC group from Cirrhosis one:

\begin{tabular}{lllllll}
\hline & \multicolumn{2}{l}{ HCC (32) } & \multicolumn{2}{l}{ Cirrhosis (8) } & Statistical test (FET) & P value \\
& No & \% & No & \% & & \\
\hline TLR4 & & & & & 1.06 & 0.24 \\
$\leq \mathbf{2 +}$ & 17 & 53.1 & 2 & 25.0 & & \\
$\mathbf{3 +}$ & 15 & 46.9 & 6 & 75.0 & & \\
AUC (95\% & 0.641 & $(0.432-0.849)$ & & & & \\
CI) & & & & & & \\
Cut off point & $2+$ & & & & & \\
Sensitivity & 53.1 & & & & & \\
Specificity & 75.0 & & & & & \\
PPV & 89.5 & & & & & \\
NPV & 28.6 & & & & & \\
Accuracy & 57.5 & & & &
\end{tabular}

AUC, area under the curve; PPV, positive predictive value; NPV, negative predictive value.

Table (8) : Validity of TLR4 to predict Cirrhosis group from hepatitis one:

\begin{tabular}{lllllll}
\hline & \multicolumn{2}{l}{ Cirrhosis (8) } & \multicolumn{2}{l}{ Hepatitis (10) } & Statistical test (FET) & P value \\
& No & $\%$ & No & $\%$ & & \\
\hline TLR4 & 2 & 25.0 & 8 & 80.0 & & 0.054 \\
$\leq \mathbf{2 +}$ & 6 & 75.0 & 2 & 20.0 & & \\
$\mathbf{3 +}$ & 6.45 & \\
AUC(95\% CI) & $0.225(0-0.456)$ & & & & \\
Cut off point & $2+$ & & & & & \\
Sensitivity & 25.0 & & & & & \\
Specificity & 20.0 & & & & & \\
PPV & 20.0 & & & & & \\
NPV & 25.0 & & & & & \\
Accuracy & 22.2 & & & & & \\
\hline
\end{tabular}

Table (9) : Validity of L-FABP to predict HCC group from Cirrhosis one:

\begin{tabular}{lllllll}
\hline & \multicolumn{2}{l}{ HCC (32) } & \multicolumn{2}{l}{ Cirrhosis (8) } & Statistical test (FET) & P value \\
& No & \% & No & \% & & 0.24 \\
\hline L-FABP & 17 & 53.1 & 2 & 25.0 & & \\
$\mathbf{1 +}$ & 15 & 46.9 & 6 & 75.0 & & \\
$\geq \mathbf{2 +}$ & 0.061 & $(0.432-0.849)$ & & & \\
AUC (95\% CI) & 0.641 & & & \\
Cut off point & $1+$ & & & & & \\
Sensitivity & 53.1 & & & & \\
Specificity & 75.0 & & & & \\
PPV & 89.5 & & & & \\
NPV & 28.6 & & & & \\
Accuracy & 57.5 & & & & \\
\hline
\end{tabular}

AUC, area under the curve; PPV, positive predictive value; NPV, negative predictive value. 
Table (10) : Validity of L-FABP to predict Cirrhosis group from hepatitis one:

\begin{tabular}{|c|c|c|c|c|c|c|}
\hline & \multicolumn{2}{|c|}{ Cirrhosis (8) } & \multicolumn{2}{|c|}{ Hepatitis (10) } & \multirow{2}{*}{$\begin{array}{l}\text { Statistical test } \\
(\text { FET) }\end{array}$} & \multirow[t]{2}{*}{$P$ value } \\
\hline & No & $\%$ & No & $\%$ & & \\
\hline L-FABP & & & & & 0.85 & 0.18 \\
\hline$\leq 2+$ & 8 & 100 & 2 & 20.0 & & \\
\hline$\overline{3}+$ & 0 & 0.0 & 8 & 80.0 & & \\
\hline $\operatorname{AUC}(95 \% \mathrm{CI})$ & 0.62 & $52-0.898)$ & & & & \\
\hline Cut off point & $2+$ & & & & & \\
\hline Sensitivity & 100 & & & & & \\
\hline Specificity & 80.0 & & & & & \\
\hline PPV & 80.0 & & & & & \\
\hline NPV & 100 & & & & & \\
\hline Accuracy & 88.9 & & & & & \\
\hline
\end{tabular}

AUC, area under the curve; PPV, positive predictive value; NPV, negative predictive value.

Table (11) : Validity of TLR4 to predict high grade HCC group from low grade one:

\begin{tabular}{llrlrll}
\hline & \multicolumn{2}{l}{ High grade (13) } & \multicolumn{2}{l}{ Low grade (19) } & Statistical test (x2) & P value \\
\hline No & \multicolumn{1}{c}{ \% } & \multicolumn{2}{c}{ No } & \% & & \\
TLR4 & 10 & 76.9 & 5 & 26.3 & 7.94 & $0.005^{* *}$ \\
$\mathbf{3 +}$ & 3 & 23.1 & 14 & 73.7 & & \\
$\leq \mathbf{2 +}$ & & & & & \\
AUC (95\% CI) & 0.753 & $(0.575-0.931)$ & & & & \\
Cut off point & $3+$ & & & & & \\
Sensitivity & 76.9 & & & & & \\
Specificity & 73.7 & & & & & \\
PPV & 66.7 & & & & & \\
NPV & 82.4 & & & & & \\
Accuracy & 75.0 & &
\end{tabular}

AUC, area under the curve; PPV, positive predictive value; NPV, negative predictive value.

Table (12) : Validity of L-FABP to predict high grade HCC group from low grade one:

\begin{tabular}{llclcll}
\hline & \multicolumn{2}{l}{ High grade (13) } & \multicolumn{2}{l}{ Low grade (19) } & Statistical test (x2) & P value \\
& No & \% & No & \% & & \\
\hline FABP1 & 9 & 69.2 & 8 & 42.1 & 2.28 & 0.13 \\
$\leq \mathbf{2 +}$ & 4 & 30.8 & 11 & 57.9 & & \\
$\mathbf{3 +}$ & & & & & \\
AUC (95\% CI) & 0.636 & $(0.438-0.833)$ & & & & \\
Cut off point & $2+$ & & & & \\
Sensitivity & 69.2 & & & & & \\
Specificity & 57.9 & & & & & \\
PPV & 52.9 & & & & & \\
NPV & 73.3 & & & & & \\
Accuracy & 62.5 & & & & & \\
\hline
\end{tabular}

$\overline{A U C}$, area under the curve; PPV, positive predictive value; NPV, negative predictive value. 

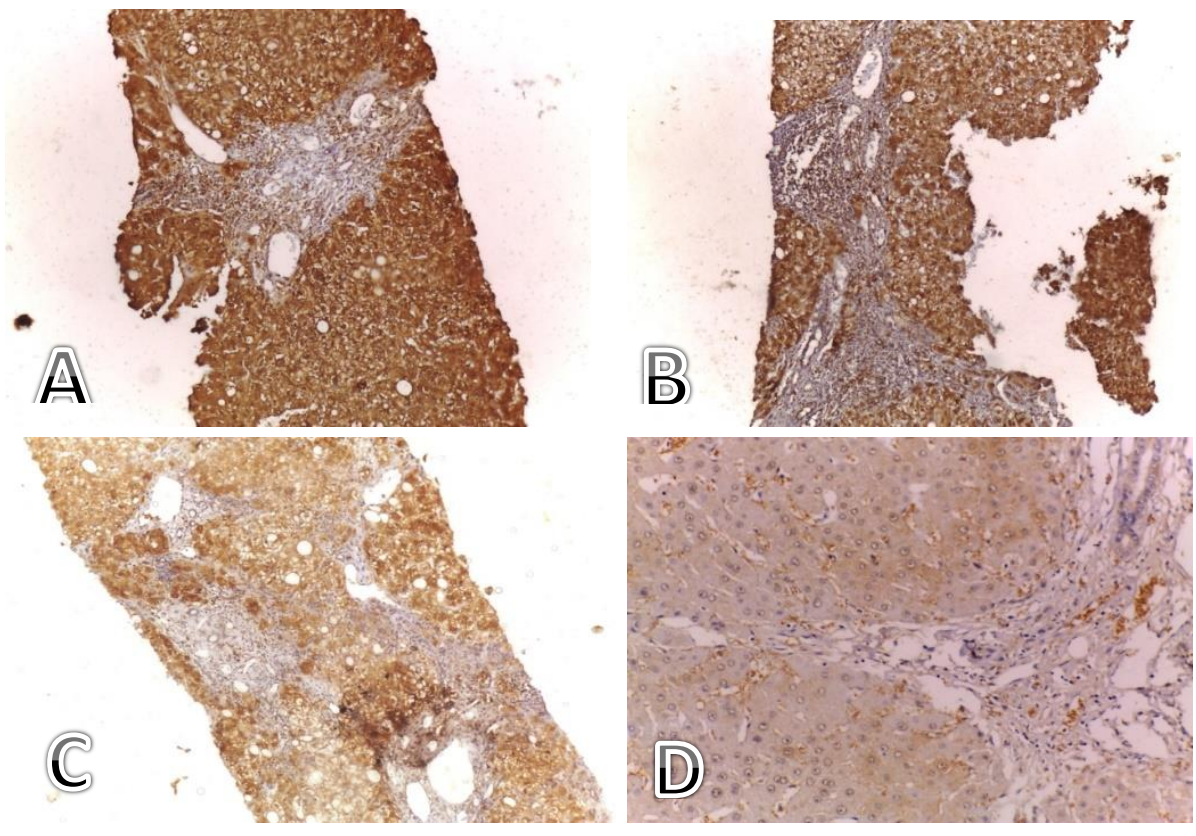

Figure ( 3):A)case of chronic hepatitis with mild activity (A1)and mild fibrosis(F1) showing positive cytoplasmic L-FABP expression score (3+) (IHC x100).B) case of chronic hepatitis with sever activity (A2)and fibrosis(F2) showing positive cytoplasmic L-FABP expression score (3+) (IHC x100).C) case of chronic hepatitis with sever activity (A3)and fibrosis(F3) showing positive cytoplasmic L-FABP expression score (2+) (IHC x100).D) case of cirrhosis showing positive cytoplasmic L-FABP expression score (2+) (IHC X200)
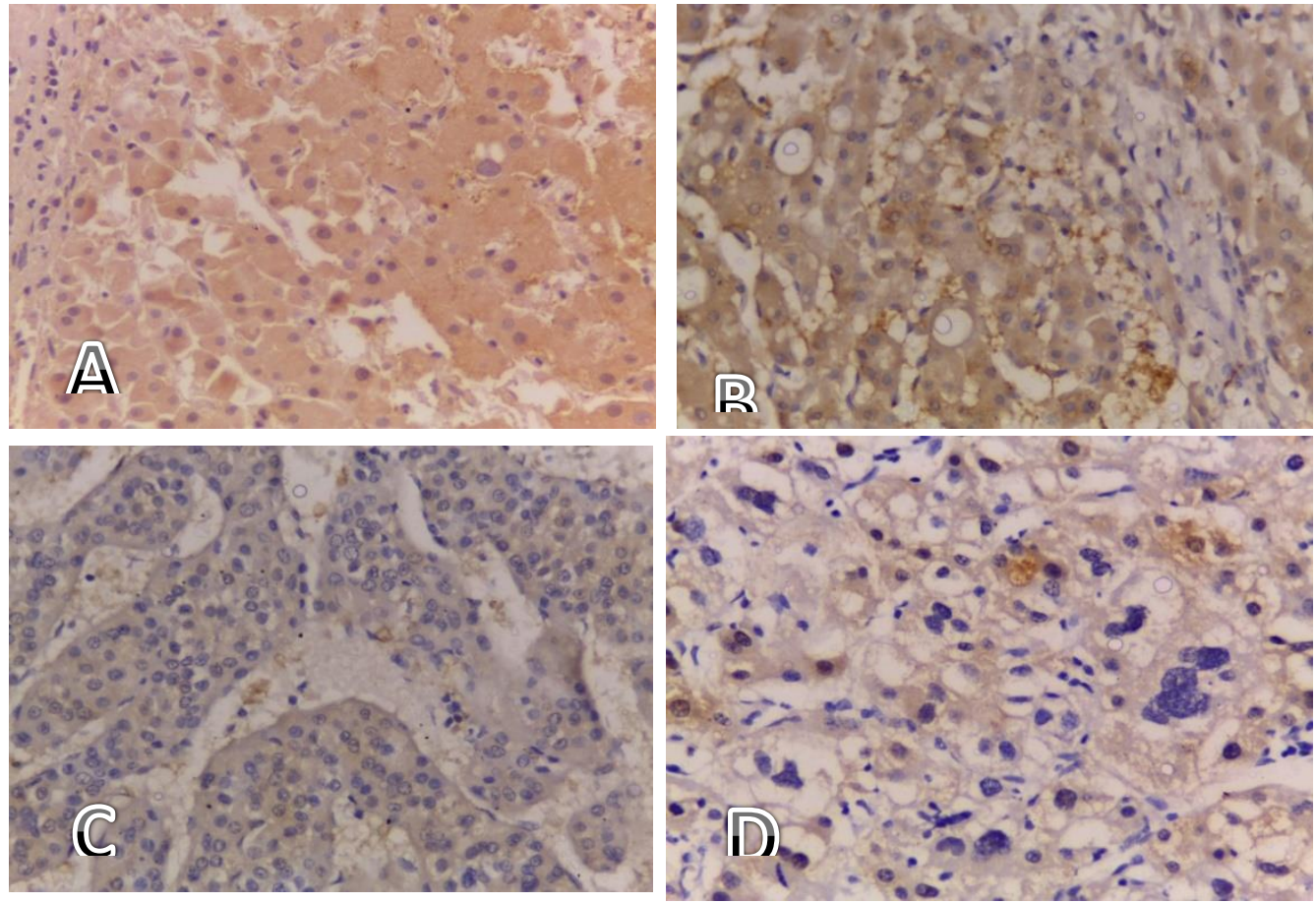

Figure(4):A) case of well differentiated HCC showing cytoplasmic L-FABP expression score (2+) (IHC $\mathbf{x 4 0 0 ) . B )}$ case of moderately differentiated HCC showed cytoplasmic L-FABP expression score (2+) (IHC $\mathbf{x 4 0 0 ) . C )}$ case of poorly differentiated HCC (grade III) showing cytoplasmic L-FABP expression score (1+) (IHC x400).D) case of undifferentiated HCC (grade IV) showing cytoplasmic L-FABP expression score (1+) (IHC x400) 



\section{Discussion:}

During recent years, evidence has been accumulating to show that inflammation has an important role in initiation, promotion, and progression of tumors .The generation of pro-inflammatory cytokines in the tumor microenvironment provokes activation of $\mathrm{NF}-\kappa \mathrm{B}$ in cancer cells, leading to protection against proapoptotic host immune defense mechanisms .It has been shown that cytokines and growth factors produced by tumor-infiltrating macrophages, lymphocytes, and other cell types in the inflammatory tumor microenvironment influence cell differentiation and exert antiapoptotic and proangiogenic effects which stimulate the growth of cancer cells, tumor invasiveness, and metastasis (18).

Hepatocellular carcinoma (HCC), a prominent example for inflammationassociated cancer, is a major complication in the end-stage of cirrhosis. In most cases, HCC in humans is the outcome of continuous injury and chronic inflammation; thus, it provides a good and realistic inflammatory-related cancer model to gain insight about the role of TLR4 in the carcinogenesis (4)
Toll-like receptor 4(TLR4) is one of the most intensively studied members of the TLR family. Lipopolysaccharides (LPS), an agonist of TLR4, induces the interaction of TLR4 with adaptor molecule MyD88, which in turn activates downstream NF-kB signaling pathways subsequently causing inflammatory mediator production and promotion of HCC cell survival and proliferation (7).

In this study, TLR4 expression in control group was detected in cytoplasm of hepatocytes score $(+1)$. This agreed with Soares et al., ( 18) who found that Under normal circumstances, the expression of TLR4 is at a relatively low level, however, when the liver is damaged under pro-inflammatory conditions , TLR4 expression is upregulated .

There was highly positive statistical significant correlation between TLR-4 expression and grade of activity, this agreed with a study in which it was proved a positive correlation between the staining scores of TLR4 expression and the grading scores in CHB. (19)

In the present study, there was positive statistical significant correlation between 
scoring of TLR-4 expression and size of HCC $(p<0.05)$. In agreement with our results two other studies (20) and (21) revealed that high expression of TLR-4 was associated with a large tumor size in breast cancer.

In the current study, there was statistical significant difference between scoring of TLR-4 expression and histopathological pattern of HCC $(p<0.05)$. This is in agreement with others who observed that TLR4 expression was significantly associated with histologic type. (22)

A positive statistical significant correlation was found between scoring of TLR-4 expression and grade of HCC ( $\mathrm{p}<$ $0.05)$, which was in concordance with others (23) who observed that TLR4 expression level was correlated with the degree of tumor differentiation and TNM stage in HCC. They explained this by TLR4/MyD88 pathway mediates the activation of NF- $\mathrm{\kappa B}$ and subsequent production of pro-inflammatory cytokines, including IL-1 $\beta$, IL-6 and TNF- $\alpha$. These cytokines stimulate myeloid dendritic cells to secret IL-23, which promotes Th17 cell differentiation, proliferation and maintenance.
Immunohistochemistry confirmed increased expression of TLR2 and TLR4 in hepatitis and cirrhosis and maintained expression in hepatocellular carcinoma as reported before (24). Upregulation of TLR2, TLR4 and their pro-inflammatory mediators is associated with virus-induced hepatic IFC sequence. Our results confirmed their findings as there was statistical significant increase in TLR-4 expression with progression from normal to chronic hepatitis $\mathrm{C}$ to cirrhosis to $\mathrm{HCC}$ group $(\mathrm{P}<0.05)$.

Liver-FABP (L-FABP) is the first of the FABPs to be described. It is bind and sequesters potentially toxic long-chain fatty acids in the cytosol so that they may be rapidly removed via oxidative or storage organelles. It is expressed in very high levels in liver, intestine and kidneys. FABP1 is positively regulated by hepatocyte nuclear factor $1(\mathrm{HNF} 1 \alpha)(9)$

As regard L-FABP expression in control group, it was detected in cytoplasm of hepatocytes score $(+3)$. In agreement with this, L-FABP showed high expression in normal colon and loss of its expression in colorectal cancer (25). 
Regarding L-FABP expression in chronic hepatitis $\mathrm{C}$ cases: L-FABP expression was were scored $(2+)$ in $20 \%$ and $80 \%$ were scored as (3+). As regard expression in cirrhosis group, $25 \%$ of cases were scored as $(1+)$ and $75 \%$ were scored as (2+) . In HCC cases, $53.1 \%$ was scored (1+), $43.8 \%$ were scored $(2+)$ and $3.1 \%$ were scored (3+). This is in agreement with other researchers, who found that expression of L-FABP is downregulated in cirrhosis and HCC. It was also observed that a greater proportion of moderately and poorly differentiated HCC showed complete loss of LFABP. It is possible that L-FABP loss may be associated with these morphologic changes and thus may represent a pathway involved in HCC differentiation/carcinogenesis (26).

In disagreement with this, it was found that Liver Fatty Acid-Binding Protein (LFABP) promotes cellular angiogenesis and migration in hepatocellular carcinoma. It was found that L-FABP was highly expressed in HCC tissues, and this expression was positively correlated with that of VEGF-A. Additionally, L-FABP significantly promoted tumor growth and metastasis in a xenograft mouse model. We also assessed the mechanisms of L-
FABP activity in tumorigenesis; L-FABP was found to associate with VEGFR2 on membrane rafts and subsequently activate pathways, which resulted in up-regulation of VEGF-A accompanied by an increase in both angiogenic potential and migration activity (27).

In the current study, there was highly statistical significant decrease in L-FABP expression with progression from normal to chronic hepatitis $\mathrm{C}$ to cirrhosis to $\mathrm{HCC}$ group $(\mathrm{P}<0.001)$. This is in agreement with a study done before (28). Moreover, this downregulation correlated with tumor differentiation and intratumoral inflammation.

These results were in disagreement with other studies (29) and (30) in which it was proved that serum Liver-Type Fatty Acid-Binding Protein is a possible prognostic factor in human chronic liver diseases, increasing from chronic hepatitis to liver cirrhosis and hepatocellular carcinoma, These difference due to their study was used on serological material and this increase in serum because its level in serum associates with tissue breakdown

This present study showed significant inverse relation between TLR4 and LFABP regarding their expression in 
chronic hepatitis $\mathrm{C}$, cirrhosis and $\mathrm{HCC}$ group $(\mathrm{P}<0.05)$. As TLR-4 expression increased with progression from normal to chronic hepatitis $\mathrm{C}$ to cirrhosis to $\mathrm{HCC}$ group. This finding coincides with these results of others (24), where it was reported increased hepatic expression of TLR2 and TLR4 in the hepatic inflammation-fibrosis-carcinoma

sequence. While L-FABP expression decreased with progression from normal to chronic hepatitis $\mathrm{C}$ to cirrhosis to $\mathrm{HCC}$ group, and agreed (28) where it was found that in HCC, L-FABP downregulation probably occurs because of phenotypic changes during tumor progression .

In this study, using receiver-operating characteristic (ROC) ,found that both TLR4 \& L-FABP were equally in accuracy to differentiate HCC from Cirrhosis , Sensitivity was $53.1 \%$, specificity was $75.0 \%$ and accuracy was $57.5 \%$, While L-FABP was more accurate than TLR4 to predict hepatitis from Cirrhosis, accuracy was $(88.9 \%$ vs $22.2 \%)$

Also by using ROC curve, TLR4 was more accurate than L-FABP to detect high grade HCC from low grade HCC, accuracy was $(75.0 \%$ vs $62.5 \%)$.
In agreement another study (31) where evaluation of patients with $\mathrm{HCV}$ related HCC by using receiver-operator characteristic (ROC) curves of TLR2 and TLR4, we found AUC of TLR2 and TLR4 $(0.72 \pm 0.06,0.77 \pm 0.05$ respectively) with high sensitivity specificity +ve Predictive value and -ve Predictive value so TLR2 and TLR4 have crucial role in pathogensis of $\mathrm{HCV}$ related $\mathrm{HCC}$ so these results raise the possibility that by targeting TLR2 and TLR4 with high affinity pharmacological stimulants may be able to control $\mathrm{HCV}$ infection by induction of IFN- $\alpha$ and direct activation of antiviral mechanisms in hepatocytes.

Additionally, they provide insight about the potential use of them as a new set of molecular markers for prognosis and outcomes of chronic HCV infection and HCC and we hope to early stage recognition of HCC make these patients eligible for potentially curative therapies, as therapeutic targets for $\mathrm{HCC}$ by inhibiting TLRs with antagonists has the potential to be a novel therapeutic technique for HCC or TLR agonists as immune tolerance.

Analysis of ROC curve of serum FABP-1 revealed that FABP-1 at cut off values (214 ng/L) sensitivity was 60\%, 
specificity was $77.5 \%$, PPV was 72.7 , NPV was $66 \%$ and AUC $=0.715$ (30). This came in agreement with another study, (32) where it was reported that when a cut-off value was $29,0 \mathrm{ng} / \mathrm{mL}$ for FABP-1, sensitivity and specificity were 75 and 100\%, respectively. Positive and negative predictive values for FABP-1 were 100 and $78 \%$, respectively (3). These results indicate that serum FABP1 can be used as a new diagnostic marker to detect liver injury and can be used in the diagnosis of chronic liver diseases, including those coupled with HCC.

\section{Conclusion:}

TLR-4 has a role in hepatocarcinogenesis as TLR-4 expression increased with progression from chronic hepatitis to cirrhosis to HCC. Increased TLR-4 expression associated with large tumor size and higher grade of $\mathrm{HCC}$, so it may serve a tool for prognosis of HCC. LFABP expression decreased from chronic hepatitis to cirrhosis to HCC. So its downregulation may contribute in pathogenesis of HCC. These may useful for the development of effective therapies that improve patient survival.

\section{References}

1- Petruzziello A. Epidemiology of hepatitis B virus $(\mathrm{HBV})$ and hepatitis $\mathrm{C}$ virus $(\mathrm{HCV})$ related hepatocellular carcinoma. Open Virol J. 2018; 12:26-32.

2- Ozakyol A. Global Epidemiology of Hepatocellular Carcinoma ,J Gastrointest Cancer. 2017; 48:238-240 .

3- Lopes Fde L, Coelho FF, Kruger JA, Fonseca GM, Araujo RL, Jeismann VB, et al . Influence of Hepatocellular Carcinoma Etiology in the Survival after Resection. Arq Bras Cir Dig. 2016; 29:105-108.

4- Refolo MG, Messa C, Guerra V, Carr BI, D'Alessandro R. Inflammatory Mechanisms of HCC Development. Cancers (Basel). 2020 Mar 10;12(3):641.

5- Zou H, Wang WK, Liu YL, Braddock M, Zheng MH, Huang DS. Toll-like receptors in hepatocellular carcinoma: potential novel targets for pharmacological intervention. Expert Opin Ther Targets. 2016 Sep; 20(9):1127-35.

6- Kiziltas S. Toll-like receptors in pathophysiology of liver diseases. World J Hepatol. 2016 Nov 18;8(32):1354-1369.

7- Liu WT, Jing YY, Yu GF, Han ZP, Yu DD, Fan QM,et al. Toll like receptor 4 facilitates invasion and migration as a cancer stem cell marker in hepatocellular carcinoma. Cancer Lett. 2015 Mar 28;358(2):136-143.

8- Wang G, Bonkovsky HL, de Lemos A, Burczynski FJ. Recent insights into the biological functions of liver fatty acid binding protein 1. J Lipid Res. 2015 Dec;56(12):223847.

9- Bioulac-Sage P, Balabaud C, Wanless I. Focal nodular hyperplasia and hepatocellular adenoma. In: Bosman FT, Carneiro F, Hruban RH, Theise ND. WHO classification of tumours of the digestive system. 4th ed. Lyon: 
International Agency for Research on Cancer, 2010: 198-204

10-Sofue K, Onoda M, Tsurusaki M, Morimoto D, Yada N, Kudo M, et al. Dual-frequency MR elastography to differentiate between inflammation and fibrosis of the liver: Comparison with histopathology. Journal of Magnetic Resonance Imaging. 2020; 51(4), 1053-1064.

11-Okabe H, Yoshizumi T, Yamashita Y, Imai K , Hayashi H, Nakagawa S, et al. Histological architectural classification determines recurrence pattern and prognosis after curative hepatectomy in patients with hepatocellular carcinoma . PloS one. 2018; 13(9).

12-Martins-Filho SN, Paiva C, Azevedo RS, Alves VAF. Histological Grading of Hepatocellular Carcinoma-A Systematic Review of Literature. Front Med (Lausanne). 2017 Nov 10;4:193.

13-Amin BM, Greene LF, Edge BS, Compton C C, Gershenwald E J, Brookland K R, et al.The Eighth Edition AJCC Cancer Staging Manual: continuing to build a bridge from a populationbased to a more "personalized" approach to cancer staging .CA Cancer J Clin 2017;67 (2):93-99

14-Li N, Xu H, Ou Y, Feng Z, Zhang Q, Zhu Q, et al. LPS-induced CXCR7 expression promotes gastric Cancer proliferation and migration via the TLR4/MD-2 pathway. Diagnostic pathology. 2019; 14(1), 3.

15-Derikx J P, Vreugdenhil A C, Van den Neucker A M, Grootjans J, van Bijnen A A, Damoiseaux J G.et al. A pilot study on the noninvasive evaluation of intestinal damage in celiac disease using I-FABP and LFABP. Journal of clinical gastroenterology. 2009; 43(8), 727-733.
16-Hayati AR, Mohamed AE, Tan GC. An immunohistochemical study of Toll-like receptors 2 and 4 in placenta with and without infection. Malaysian J Pathol 2010; $32(1): 13-19$.

17-Ku CY, Liu YH, Lin HY, Lu SC, Lin JY. Liver Fatty Acid-Binding Protein (L-Fabp) Promotes Cellular Angiogenesi And Migration In Hepatocellular Carcinoma. Oncotarget.2016;7(14):18229-18245

18-Soares JB, Pimentel-Nunes P, RonconAlbuquerque R, Leite-Moreira A. The role of lipopolysaccharide/toll-like receptor 4 signaling in chronic liver diseases. Hepatol Int. 2010 Oct 21;4(4):659-72.

19-Wei XQ, Guo YW, Liu JJ, Wen ZF, Yang SJ, Yao JL. The significance of Toll-like receptor 4 (TLR4) expression in patients with chronic hepatitis B. Clin Invest Med. 2008;31(3):E12330.

20-González-Reyes S, Marín L, González L, González LO, del casar JM, lamelas ML. Study of Tlr3, Tlr4 and Tlr9 in breast carcinomas and their association with metastasis. BMc cancer. 2010; 10: 665 .

21-Shi S, Xu C, Fang X, Zhang Y, Li H, Wen W, et al. Expression profile of Toll-like receptors in human breast cancer. Mol Med Rep. 2020 Feb;21(2):786-794.

22-Wang EL, Qian ZR, Nakasono M, Tanahashi T, Yoshimoto K, Bando Y, et al. High expression of Toll-like receptor 4/myeloid differentiation factor 88 signals correlates with poor prognosis in colorectal cancer. $\mathrm{Br} \mathrm{J}$ Cancer. 2010 Mar 2;102(5):908-15.

23-Kang Y, Su G, Sun J, Zhang Y. Activation of the TLR4/MyD88 signaling pathway 
contributes to the development of human hepatocellular carcinoma

via upregulation of IL-23 and IL-17A. Oncol Lett. 2018 Jun;15(6):9647-9654.

24-Soares JB, Pimentel-Nunes P, Afonso L, Rolanda C, Lopes P, Roncon-Albuquerque R Jr. Increased hepatic expression of TLR2 and TLR4 in the hepatic inflammation-fibrosiscarcinoma sequence. Innate Immun. 2012 Oct; $18(5): 700-8$.

25-Lawrie LC, Dundas SR, Curran S, Murray GI. Liver fatty acid binding protein expression in colorectal neoplasia. Br J Cancer. 2004 May 17;90(10):1955-60.

26-Cho SJ, Ferrell LD, Gill RM. Expression of liver fatty acid binding protein in hepatocellular carcinoma. Hum Pathol. 2016 Apr;50:135-9.

27-Ku CY, Liu YH, Lin HY, Lu SC, Lin JY. Liver Fatty Acid-Binding Protein (L-Fabp) Promotes Cellular Angiogenesi And Migration In Hepatocellular Carcinoma. Oncotarget. 2016;7(14):18229-18245

28-Inoue M, Takahashi Y, Fujii T, Kitagawa M, Fukusato T. Significance of downregulation of liver fatty acid-binding protein in hepatocellular carcinoma. World J Gastroenterol. 2014 Dec 14;20(46):17541-51.
29-Eguchi A, Hasegawa H, Iwasa M, Tamai Y, Ohata K, Oikawa T et al . Serum Liver-Type Fatty Acid-BindingProtein Is aPossible Prognostic Factor in Human Chronic Liver Diseases From Chronic Hepatitis to Liver Cirrhosis and Hepatocellular Carcinoma HepatologyCommuniCations . 2019 ; 39(6):825- 830 .

30-Albadawy R M, Abd El-Aziz B A, Behiry E G, Azzam M E. Assessment the Association of Fatty Acid Binding Protein 1 with Hepatocellular Carcinoma. Benha Medical Journal. 2020 January 19.

31-Hussein R M, Abd El-Halim H, Shatat M, Allam E, Abd El-Gahny W, Tawfik H M. Relation Of Toll Like Receptor 2 And 4 To HCC Parameters . Research Journal of Pharmaceutical, Biological and Chemical Science.2019;10(3):469.

32-Akbal E, Köklü S , Koçak E, Çakal B, Güneş F , Başar O. Liver Fatty Acid-Binding Protein Is A Diagnostic Marker To Detect Liver Injury Due To Chronic Hepatitis C Infection. Official Journal Of The Institute Mexicano Del Seguro Social. 2013; 44 (1): 34.38.

To cite this article: Eman El-Gendy, Reda El badawy, Ranih Zakaria, Taghreed Abd Elsamee, Hala A. Agina. Significance of Toll-like Receptor 4 (TLR4) \& Liver Fatty Acidbinding Protein (L-FABP) Expression in Hepatocellular Carcinoma : An Immunohistochemical Study. BMFJ 2021;38 (academic issue): 92-111, DOI: 10.21608/bmfj.2020.52389.1352 